\title{
Towards a Dependable Cardiovascular Surveillance System
}

\author{
Cristina C. Oliveira ${ }^{1}$ and José Machado da Silva ${ }^{1}$ \\ ${ }^{1}$ INESC TEC, Faculdade de Engenharia, Universidade do Porto, Portugal
}

\begin{abstract}
An aneurysm is a life-threatening condition, which left untreated may burst or rupture, causing massive blood loss. One of the currently available treatments for aortic aneurysms is endovascular aneurysm repair (EVAR). However, in spite of major advances in the surgical techniques, complications are still likely to occur making it recommendable to maintain lifelong surveillance. In order to reduce and even eliminate current surveillance imaging exams, as well as to reduce followup costs, new technological solutions are being pursued. The work presented herein aims to develop a novel and dependable non-invasive coronary stent-graft monitoring system based on RFID technology. The monitoring system uses an inductive coupling interface to capture the pressures given by a cluster of sensors placed on the stent-graft's wall. The application of multimodal data fusion techniques enables the improvement of the surveillance system performance in terms of accuracy, robustness and reliability. The processing of the pressure signals measured inside the aneurysm sac with other physiological signals electrocardiogram (ECG) and arterial blood pressure (ABP) will allow obtaining better monitoring resolution and reliability. The techniques utilized to diagnose deviations from the normal operation or faults in the flexible pressure sensors are described and tested, showing promising results for achieving a highly reliable system.
\end{abstract}

Keywords - dependability, cardiovascular surveillance, fault detection, inductive coupling, pressure sensors.

\section{INTRODUCTION}

Life expectancy has increased in developed countries, namely in European Union and North America, demanding for effective and safe treatments of diseases of the cardiovascular treat. The abdominal aortic aneurysm (AAA) is a dangerous pathology with high rate of occurrence among elderly people. When timely detected, most AAA can be repaired through either open thoracic surgical intervention or endovascular aneurysm repair (EVAR). The latter involves significant less morbidity risks and consists of a minimally invasive procedure in which a stent-graft is guided from the femoral artery to the affected artery segment. This treatment requires posterior regular surveillance in order to detect and prevent complications such as endoleaks. The main current postEVAR surveillance method is radiation based imaging (computed tomography and magnetic resonance) of the repaired region and geometric measurement of the aneurysm sac. This is expensive, uncomfortable and not safe to the patient [1]. To increase safety and effectiveness in post-EVAR monitoring, a single sensor wireless pressure-monitoring system has been recently developed with encouraging preliminary results [2].

Medical application systems demand high levels of dependability and trust of performance [3]. According to the IEC (International Electrotechnical Commission) IEV (International Electrotechnical Vocabulary) 191-02-03 standard "dependability (is) the collective term used to describe the availability performance and its influencing factors: reliability performance, maintainability performance and maintenance support performance". A system's dependability study includes the attributes analysis (e.g., availability, security and maintainability), risks (defects and failures), means (of prevention, detection and fault tolerance) and enables the objective of an efficient development of test and self-test methodologies. For implantable medical devices safety is a key element, since according to the European Council Directive 90/385/EEC on the approximation of the laws of the member states relating to active implantable medical devices [4] "The devices must be designed and manufactured in such a way that, when implanted (...) their use does not compromise the clinical condition or the safety of patients. They must not present any risk to the persons implanting them or, where applicable, to other persons". A dependability analysis can be conducted at a system level or at different levels, such as analog circuits, software and/or network levels, each one requiring a specific approach in order to increase dependability. The development of an implantable pressure sensor requires a cross-disciplinary team where physicians, surgeons, micro-electronics and mechanics specialists work together in order to develop an implant with the highest dependability level. The first step on the development of a dependable medical monitoring device is identifying the system's risks of not achieving acceptable levels of dependability. After this, fault detection and fault tolerance/reconfiguration techniques will be used to detect system malfunctions and correct them in order to achieve high levels of dependability [5],[6]. Data fusion techniques can also be utilized to improve the cardiovascular surveillance system in mainly two aspects. The first is to increase the trustiness of the captured bio signals, after rejecting or correcting the corrupted ones, and the second is to 
provide additional information on the patient's condition by classifying the set of signals into normal or abnormal condition (e.g. arrhythmia, chest angina, stroke, etc) [7],[8].

A summary of the cardiovascular surveillance system under development is presented in section II. Section III describes the pressure sensors' diagnostic and fault detection approach being proposed and some experimental results. Section IV presents the status of the work and main challenges.

\section{SYSTEM DESCRIPTION}

Our aim is to develop a combined cardiac and aortic surveillance system, comprising a wearable cardiac monitor and an advanced telemetry system for post-EVAR surveillance (figure 1). Its main innovation resides in the synchronous measurement of the patient electrocardiogram (ECG) and intra-sac aneurysm pressure, in order to have a more robust and reliable monitoring.

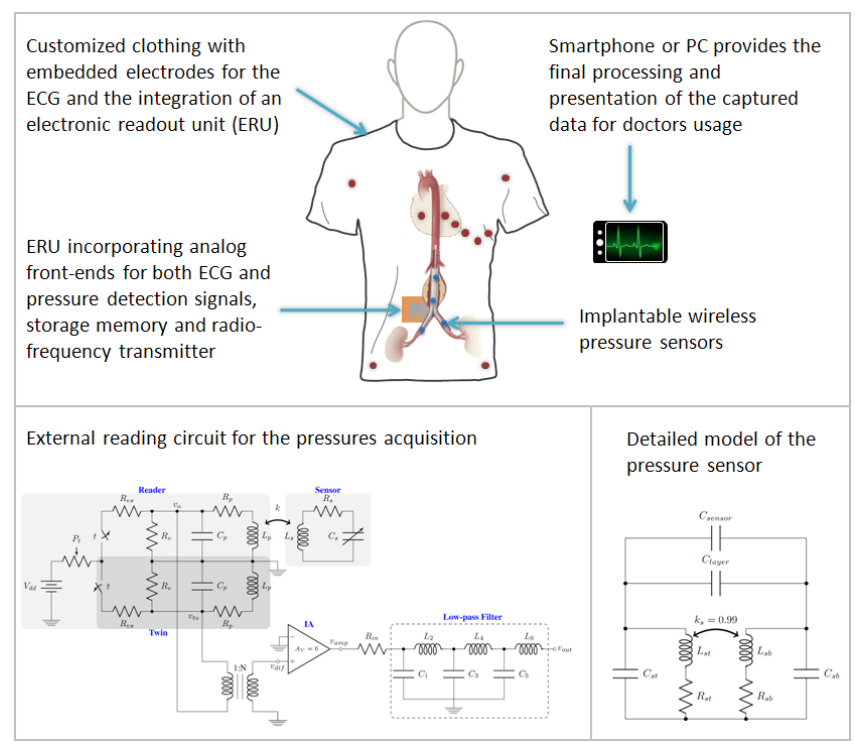

Fig. 1: Wearable integrated cardiovascular surveillance system.

For that purpose implantable wireless pressure sensors which are biologically compatible and show suitable linearity and sensitivity were produced by Sepúlveda et. al [9]. An electronic readout unit (ERU) capable of energizing the pressure sensors and capture the pressure data was also developed and tested, proving to be capable of energizing an $L C$ resonant circuit (with the same electrical characteristics of the pressure sensor) and capture the respective oscillation frequency with good accuracy [10],[11].

Our purpose is to embed the ECG electrodes and the ERU, in order to capture both the ECG and the intra-sac aneurysm pressure, into a customized clothing to achieve a system with high levels of dependability. With that aim a system based on sensor and data fusion, as well as the development of circuits that comprise fault detection and built-in self-test modalities, is proposed. This raised the need, on the one hand, to combine strategies and techniques addressing the development of dependable medical systems, and, on the other hand, the adoption of very low power consumption and intrinsic lownoise design techniques in order to implement the system on a monolithic support using a MOS technology. Since medical application systems demand high levels of dependability and trust of performance, the processing circuits should satisfy demanding testability requirements.

The flexible capacitive pressure sensors are fabricated using aligned carbon nanotubes (A-CNTs) embedded in a flexible substrate of polydimethylsiloxane (PDMS), a transparent, nontoxic and biocompatible silicone elastomer, forming a dielectric which is hermetically sealed at ambient pressure) [9]. The antennas that comprise the capacitive sensors were fabricated using DuPont ${ }^{\mathrm{TM}}$ Kapton ${ }^{\circledR}$ polyimide film in two different geometries (square and circle). For each geometry, three sizes antennas were fabricated in order to test different dielectrics (pressure sensor membrane): $4 \mathrm{~mm}, 6 \mathrm{~mm}$ and 8 $\mathrm{mm}$. The antennas are built with two stacked inductors, one on each side of the polyimide film, interconnected with a via on the outer segment of each.

Since the developed sensor has a new structure, which is different from conventional CMOS or Printed Circuit Boards (PCB) RFID antennas, an electrical model was developed (see right bottom side of figure 1). Each layer forms a resonant circuit comprising an inductance $L_{s}$, the series resistance $R_{S}$ and the parasitic capacitance $C_{S}$ due to the capacitance formed between the traces of the inductor. A parasitic capacitance $C_{\text {layer }}$, formed between the traces of the top and bottom layers, is also connected between the layers in parallel, as well as the sensor's capacitance $C_{\text {sensor }}$.

Table 1 presents calculated parameters for a square pressure sensor with a dieletric of $4 \mathrm{x} 4 \mathrm{~mm}$. The expressions for the calculations of planar spiral inductance presented in [12] were used for the estimation of the inductance. The total inductance of the dual-layer planar inductor is almost 4 times higher than a single layer inductor with the same dimensions $L_{\text {total }} \cong L_{s t}+L_{s b}+2 M$. By design the current flows in the same direction on both layers, plus the proximity and the via between the inductors assures the mutual inductance $M$ to be almost equal to $L_{s}\left(M=k_{s} \sqrt{L_{s t} L_{s b}} \cong L_{s}\right)$. The sensor's capacitances were estimated using the expression for the parallel plate capacitor.

The performance of the flexible sensor to different pressures, and its use with the telemetry system, were evaluated 
Table 1: Sensor parameters.

\begin{tabular}{c|c|c|c|c}
\hline$L_{S}[\mu \mathrm{H}]$ & \multicolumn{1}{c}{$R_{S}[\Omega]$} & $C_{s}[\mathrm{pF}]$ & $C_{\text {layer }}[\mathrm{pF}]$ & $C_{\text {sensor }}[\mathrm{pF}]$ \\
\hline \hline 5.41 & 3.99 & 2.31 & 33.63 & 2.83 \\
\hline
\end{tabular}

using a vacuum chamber. Although the blood pressure in the aneurysm sac ranges from $20 \mathrm{mmHg}$ to $250 \mathrm{mmHg}$ referring to atmospheric pressure (1 atm to $1.3 \mathrm{~atm})$ [13], the pressure sensors were only tested for pressures between $0 \mathrm{~atm}$ and $1 \mathrm{~atm}$ (vacuum to atmospheric pressure) due to technological constraints. The output of the reader circuit was recorded while commercial pressure sensors placed also inside the chamber were used to record reference values for comparison purposes. Results from the measurements with pressure sensor are presented in Figure 2.

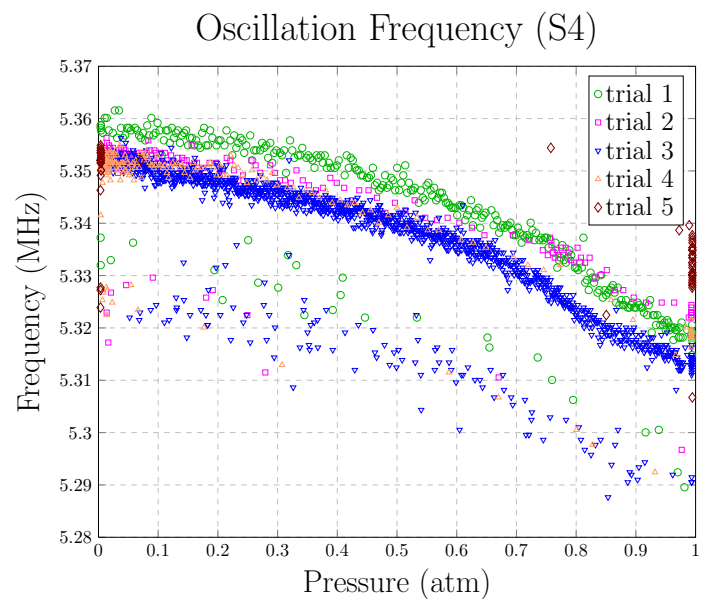

Fig. 2: Measurements in the vacuum chamber.

The slope of variation of the oscillation frequency with pressure $\left(\frac{d f_{\text {osc }}}{d P}\right)$ is not constant, but its sign doesn't change, meaning that an increase in the pressure - and thus of the sensing capacitor - always corresponds to a decrease of the oscillation frequency. The characteristic in figure 2 shows that the sensor's best sensitivity would be that in the range of 0.8 atm to $1 \mathrm{~atm}$ (the behavior is similar in the $1 \mathrm{~atm}$ to $1.2 \mathrm{~atm}$ pressure range) meaning that, for the sake of the proposed application, the $L C$ sensor network shows the highest sensitivity in the range of interest.

Moreover, the variation rate is several times larger than the mean deviation from the trend-curve, which shows that the obtained transfer characteristic provides good sensitivity. Small variations between different trials also suggest good precision. The high pressure difference (between the dielectric and external pressure) that the sensors experience during the trials $(\triangle \mathrm{P}=1 \mathrm{~atm})$ validate the good sealing characteris- tics of the bonding process used and the high dynamic range of the sensors.

The pressure sensors are being subjected to preliminary tests focused on the characterization and performance, and so far no tests in vitro or in vivo were performed.

\section{SENSOR Diagnostics}

Regarding the wearable integrated cardiovascular surveillance system under development, one of the objectives in our work is to detect faults occurring in the sensors and in the circuitry which would rend misinterpretations of the patient health condition. The occurrence of defects in the $L C$ sensor circuit may mask the detection of anomalies in the stentgraft. For example, a reduction of capacitor's measurement range would still allow detecting pressure deviations but these could be taken as good even if the patient condition had deteriorated; a large deviation of capacitor's nominal value could lead to a false defective stent-graft detection (e.g. a leaking stent-graft); a capacitor stuck at a constant value would provide an anomalous constant pressure.

The telemetry system is being improved in order to allow detecting faults or large deviations in the sensor. For this purpose one takes advantage of the ECG signal to provide the fault detection triggering signal, of the measurement of the power transmission from the sensor to the reader circuit, as well as of the reflected impedance [14].

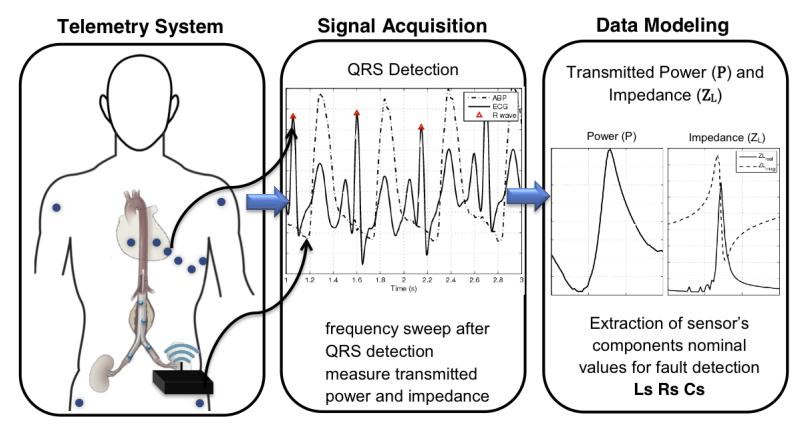

Fig. 3: Fault detection block diagram.

Simulations were performed in order to estimate the power transmission and the reflected impedance of the sensors using the detailed sensor electric model in figure 1 and the fault detection circuit described in [14]. Figure 3 displays the curves for power and impedance of a sensor. This model predicts well the small resonances around the expected sensor's main frequency. The simulation results show to be in good agreement with measurements obtained in the lab. 
Nevertheless, for diagnosis purposes, the simple $L C$ sensor model will still be used to extract the values for $L_{\text {stotal }}$ and $C_{\text {stotal }}$, since there are only 2 measurements (power and impedance) for the estimation of 2 parameters. These extracted values are related with the simple sensor electric model from Figure 1 in the following way: $L_{s t o t a l}=4 L_{s}$, $R_{\text {total }}=2 R_{S}$ and $C_{\text {stotal }}=C_{\text {layer }}+C_{\text {sensor }}$.

Table 2 depicts the calculated and extracted values for the pressure sensor using the fault detection algorithm to measure the power and impedance, and fit these curves to find the sensor's parameter values. As expected, the $R_{\text {total }}$ and $C_{\text {stotal }}$ extracted values are higher than calculations, due to the fact that during the assembly of the A-CNTs onto the flex PCB using a special conductive glue, extra parasitic capacitance and resistance are inserted, which cannot be calculated. The antenna's inductance was measured with a network analyzer prior to the assembly of the A-CNTs. The measured inductance is $18.08 \mu \mathrm{H}$, which is lower than the extracted value. However the antenna is not purely inductive, since the overlap of the top and bottom coils introduces a significant series capacitance, which causes the difference in the inductance value.

Table 2: Calculated and extracted (data fitting using power and impedance curves measured with the fault detection circuit) parameters for the sensor.

\begin{tabular}{c|c|c}
\hline Parameters & Calculated & Extracted \\
\hline \hline$L_{\text {stotal }}[\mu \mathrm{H}]$ & 22.17 & 21.90 \\
$C_{\text {stotal }}[\mathrm{pF}]$ & 36.47 & 44.98 \\
$R_{\text {total }}[\Omega]$ & 7.98 & 20.38 \\
\hline
\end{tabular}

This testing procedure can also be used to characterize the sensors after the fabrication process, since the deposition of the PDMS layer to protect the sensor and make it bio compatible can change some parameters, like the resistance and capacitance, and prevents electrical access to probing points once it is totally coated.

\section{CONCLUSION}

A dependable monitoring system for wireless detection of endoleaks in coronary stent-grafts is being developed. A methodology to diagnose deviations in the $L C$ values after measurements of the power and impedance seen from the reader circuit has been developed using a simple data fusion approach. Other data fusion techniques are being explored to further improve the cardiac and coronary surveillance system in order to achieve a highly reliable system.

\section{CONFLICT OF INTEREST}

The authors declare that no conflicts of interest of any nature are involved in this work.

\section{ACKNOWLEDGEMENTS}

The authors acknowledge the support of the ERDF - European Regional Development Fund through the COMPETE Programme (operational programme for competitiveness) and National Funds through the FCT Fundação para a Ciência e a Tecnologia (Portuguese Foundation for Science and Technology) within project SIVIC PTDC/EEI-ELC/1838/2012 (FCOM01-0124-028937) and grant contract SFRH/BD/81476/2011.

\section{REFERENCES}

1. The United Kingdom EVAR Trial Investigators . Endovascular versus Open Repair of Abdominal Aortic Aneurysm New England Journal of Medicine. 2010;362:1863-1871.

2. Ohki T, Ouriel K, Silveira PG, et al. Initial results of wireless pressure sensing for endovascular aneurysm repair: The APEX Trial-Acute Pressure Measurement to Confirm Aneurysm Sac EXclusion Journal of Vascular Surgery. 2007;45:236-242.

3. Council directive 93/42/EEC of 14 June 1993 concerning medical devices 1993.

4. Council directive 90/385/EEC of 20 June 1990 on the approximation of the laws of the Member States relating to active implantable medical devices 1990 .

5. Le Floch F, Bernard S, Bontorin G, Soulier F, Cathebras G. Global strategy to guaranty dependability of electrical medical implanted devices in 2011 5th International IEEE/EMBS Conference on Neural Engineering (NER):515-518 2011.

6. Cathebras G, Le Floch F, Bernard S, Soulier F. Dependability: a challenge for electrical medical implants in 2010 Annual International Conference of the IEEE Engineering in Medicine and Biology Society $(E M B C): 5923-59262010$.

7. Kenneth E, Rajendra AU, Kannathal N, Lim CM. Data Fusion of Multimodal Cardiovascular Signals in Engineering in Medicine and Biology Society, 2005. IEEE-EMBS 2005. 27th Annual International Conference of the:4689-4692 2005.

8. Alemzadeh H, Jin Z, Kalbarczyk Z, Iyer RK. An embedded reconfigurable architecture for patient-specific multi-paramater medical monitoring in 2011 Annual International Conference of the IEEE Engineering in Medicine and Biology Society,EMBC:1896-1900 2011.

9. Sepúlveda AT, Villoria R, Viana JC, Pontes AJ, Wardle BL, Rocha LA. Full elastic constitutive relation of non-isotropic aligned-CNT/PDMS flexible nanocomposites Nanoscale. 2013;5:4847-4854. PMID: 23616092.

10. Oliveira C, Almeida N, Machado da Silva J. Inductive coupling system for endovascular aneurysm repair monitoring Studies in health technology and informatics. 2012;177:101-106.

11. Oliveira C, Almeida N, Machado da Silva J. A stent-graft endoleakage monitor: Telemetry system based on inductive-coupling transmission for implantable pressure sensors in Bioengineering (ENBENG), 2013 IEEE 3rd Portuguese Meeting in:1-4 2013.

12. Mohan SS, del Mar Hershenson M, Boyd SP, Lee TH. Simple accurate expressions for planar spiral inductances Solid-State Circuits, IEEE Journal of. 1999;34:14191424.

13. Potkay J. Long term, implantable blood pressure monitoring systems Biomedical Microdevices. 2008;10:379-392.

14. Oliveira C, Machado da Silva J. Fault Detection System for a StentGraft Endoleakage Monitor in Mixed-Signals, Sensors and Systems Test Workshop (IMS3TW), 2012 18th International:17-21 2012. 